\title{
On the nature of blob propagation and generation in the Large Plasma Device: Global GRILLIX studies
}

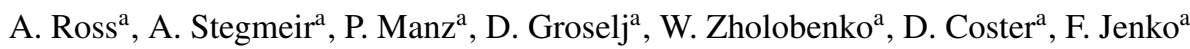 \\ ${ }^{a}$ Max-Planck-Institut für Plasmaphysik, D-85748 Garching, Germany
}

\begin{abstract}
The appearance of blobs, filamentary structures featuring large intermittent perturbations, is characteristic for the scrape-off layer (SOL) of magnetic fusion devices. Therefore, a global model, which does not rely on assumptions about the fluctuation level, is necessary to model blobs accurately. Whereas GRILLIX, a global 3D fluid turbulence code, is originally designed to handle complex geometries via the flux-coordinate independent approach (FCI), the analysis here aims on a thorough verification, validation and identification of basic phenomena in simplified slab geometry. As such the impact of the routinely employed Boussinesq approximation is studied systematically, revealing that not only the density amplitude itself matters, but also the blob regime that is also influenced by temperature. This points out that the validity of the Boussinesq approximation cannot generally be taken for granted. Furthermore, GRILLIX is validated against the LAPD experiment and the formation of blobs is studied. A cross-phase evaluation suggests as candidates for the blob drive mechanism the rotational iterchange instability.
\end{abstract}

Keywords: Blobs, Scrape-off layer (SOL), Turbulence, Validation, Large Plasma Device (LAPD), Boussinesq approximation

\section{Introduction}

Radial transport of plasma in the edge and scrape-off layer (SOL) of magnetically confined plasmas is highly turbulent. This turbulence is mainly dominated by filamentary largeamplitude structures in density, electron temperature and electric potential known as 'blobs' [1, 2, 3, 4]. It is known from theory and simulation that these blobs are born as a result of the nonlinear saturation of the underlying edge turbulence instabilities [5]. These coherent structures are spatially localized in the poloidal plane on a lower-density plasma background and are elongated along the magnetic field lines. A comprehensive review of blob physics is given in $[6,7,8]$. Analytical theories, which are able to describe the basic blob dynamics were derived in $[9,10,11,12]$, but most of the numerical studies were performed in $2 \mathrm{D}[13,5,14,15,16]$. However, blobs are a full $3 \mathrm{D}$ phenomenon such that in recent years the new standard for blob dynamics became 3D simulations [17, 18, 19, 20, 21, 22]. The numerical results of blob dynamics can be compared to the analytical scaling laws.

Reliable modelling of the tokamak scrape-off layer is crucial for the realization of a future fusion device. The correct description of the blob dynamics is important in this context since plasma turbulence in the SOL consists mainly of blobs, which determine the profiles, the SOL width and the heat loads on the divertor plates. Several code projects like GBS [23, 24], HERMES(BOUT++)[25], TOKAM3X [26], GDB [27] and GRILLIX [28, 29] aim to simulate SOL turbulence self consistently based on 3D Braginskii fluid models [30, 31].

Email address: Alexander. Ross@ipp.mpg.de ()
GRILLIX employs the drift reduced Braginskii equations [30] described in section 2. The implemented model is global, as it retains the full parametric dependencies and does not make use of the routinely Boussinesq approximation. GRILLIX is based on the flux-coordinate independent approach (FCI) $[32,33]$, which allows the treatment of complex diverted geometries [28, 29]. However, we employ here GRILLIX in a simplified slab geometry. This allows an investigation of basic phenomena, accurate verification and validation, which is often difficult in complex geometries. We study blob propagation in section 3 and verify the implementation of GRILLIX based on analytically derived scaling laws. Furthermore, we investigate the impact of the Boussinesq approximation systematically across different blob regimes. In section 4 we perform simulations for the linear device LAPD [34]. We clarify the origin of blob formation via evaluating the cross-phase between the density and electrostatic potential and comparing it to the characteristics of the relevant plasma instabilities. Finally, experimental data from $[35,36]$ allows to perform a validation of GRILLIX vs. the LAPD experiment.

\section{The GRILLIX code}

\subsection{Slab geometry}

The GRILLIX code is based on the flux-coordinate independent approach (FCI) that is very flexible with respect to geometry and can deal with realistic diverted geometries [28, 29]. Here, we use GRILLIX in a simplified slab geometry to study blob propagation in a flux tube and turbulence in the linear device LAPD. Otherwise the same algorithms and numerical techniques as in the full FCI version are used. 


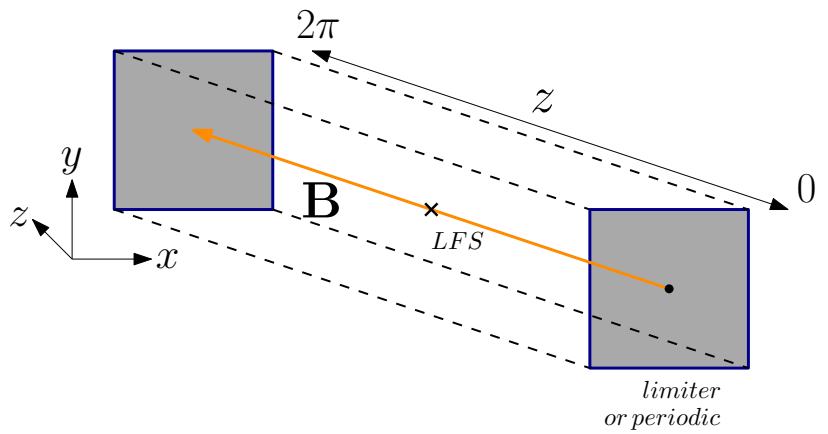

Figure 1: Slab geometry: The magnetic field is directed along $z$, where Bohmsheath boundary conditions are applied at $z=0$ and $z=2 \pi$. In flux tube mode an effective curvature is present.

The simulation domain is a rectangular box $(x, y, z)$ with $z$ a coordinate along the magnetic field $\mathbf{B}=B_{0} \hat{z}$ and $x, y$ coordinates perpendicular to the magnetic field (see Fig. 1). At the ends of the box - in normalised coordinates at $z=0$ and $z=2 \pi$ - Bohm-Sheath boundary conditions (see Eq. 9) are applied modelling contact of the plasma with target plates. The box is periodic in $y$ and Dirichlet or Neuman boundary conditions are provided for the dynamical fields in $x$ direction. Provided that the box is sufficiently large, the results are independent of the perpendicular boundaries, as the main loss mechanism is along magnetic field lines. In addition to strictly linear devices, an unwound flux tube in the SOL of tokamaks can be modelled via an effective curvature operator. The flux tube ranges between the limiter or divertor plates and effects of magnetic shear are not captured. The effective curvature operator is defined as:

$$
C(f):= \begin{cases}0, & \text { in linear mode } \\ -2\left(\sin \left(z-z_{0}\right) \frac{\partial f}{\partial x}+\cos \left(z-z_{0}\right) \frac{\partial f}{\partial y}\right), & \text { in flux tube mode }\end{cases}
$$

where via the parameter $z_{0}$ the course of curvature with respect to the sheath boundaries can be controlled, i.e. for $z_{0}=0$ the position of unfavourable curvature is located at $z=\pi$.

\subsection{Physical model}

The drift reduced Braginskii equations [31, 30] are employed to model turbulence in LAPD. At electron temperatures of typically $T_{e 0} \sim 6 \mathrm{eV}$, densities of $n_{0} \sim 2 \times 10^{12} \mathrm{~cm}^{-3}$ and magnetic field strengths of $B_{0}=0.1 \mathrm{~T}[34,35]$, the assumptions of a fluid approach are usually well fulfilled. Furthermore, the cold ions are assumed based on the assumption $T_{i 0} \ll T_{e 0}$ $\left(T_{i 0} \sim 1 \mathrm{eV}\right.$ in LAPD). Perpendicular coordinates $(x, y)$ are normalised against the sound Larmor radius $\rho_{s 0}=c \sqrt{T_{e 0} M_{i}} /\left(e B_{0}\right)$, parallel scales against the connection length $L_{\|} /(2 \pi)$ and time against $L_{\|} /\left(2 \pi c_{s 0}\right)$ with sound speed $c_{s 0}=\sqrt{T_{e 0} / M_{i}}$. The dynamical fields evolved in GRILLIX are density $n$, electron temperature $T_{e}$ normalised to reference values $n_{0}$ and $T_{e 0}$, electrostatic potential $\phi$ normalised to $T_{e 0} / e$, parallel ion velocity $u_{\|}$ normalised to $c_{s 0}$, parallel current $j_{\|}$normalised to $e n_{0} c_{s 0}$ and parallel component of perturbed electromagnetic vector potential $A_{\|}$normalised to $\beta_{0} B_{0} \rho_{s 0}$ with $\beta_{0}=4 \pi n_{0} T_{e 0} / B_{0}^{2}$ the dynamical plasma beta. The set of equations implememted in GRIL-
LIX can be written in slab geometry as:

$$
\begin{aligned}
& \frac{d}{d t} n=-\partial_{z}\left(n v_{\|}\right)+n C(\phi)-C\left(p_{e}\right), \\
& \nabla \cdot\left[n\left(\frac{d}{d t}+u_{\|} \partial_{z}\right) \nabla_{\perp} \phi\right]=-C\left(p_{e}\right)+\partial_{z} j_{\|}, \\
& \left(\frac{d}{d t}+u_{\|} \partial_{z}\right) u_{\|}=-\frac{1}{n} \partial_{z} p_{e}, \\
& \beta_{0} \frac{\partial}{\partial t} A_{\|}+\mu\left(\frac{d}{d t}+v_{\|} \partial_{z}\right) \frac{j_{\|}}{n}=-\left(\frac{\eta_{\| 0}}{T_{e}^{3 / 2}}\right) j_{\|} \\
& \quad-\partial_{z} \phi+\frac{1}{n} \partial p_{e}+0.71 \partial_{z} T_{e}, \\
& \frac{3}{2}\left(\frac{d}{d t}+v_{\|} \partial_{z}\right) T_{e}=T_{e} C(\phi)-\frac{T_{e}}{n} C\left(p_{e}\right)-\frac{5}{2} T_{e} C\left(T_{e}\right) \\
& \quad+0.71 \frac{T_{e}}{n} \partial_{z} j_{\|}+\left(\frac{\eta_{\| 0}}{T_{e}^{3 / 2}}\right) \frac{j_{\|}}{n}+\frac{1}{n} \partial_{z}\left[\left(\chi_{\| e 0} T_{e}^{5 / 2}\right) \partial_{z} T_{e}\right], \\
& \nabla_{\perp}^{2} A_{\|}=-j_{\|},
\end{aligned}
$$

where we defined as auxiliary variables the pressure $p_{e}=n T_{e}$ and the parallel electron velocity $v_{\|}=u_{\|}-j_{\|} / n$. Advection with $E \times B$ velocity is contained in $\frac{d}{d t} f=\partial_{t} f-\delta_{0}\left(\partial_{x} \phi \partial_{y} f-\partial_{y} \phi \partial_{x} f\right)$. Equations (2)-(8) are the continuity equation, vorticity equation, parallel ion momentum equation, Ohm's law, electron temperature equation and Farayday's law. The dimensionless parameters of the system are the drift scale $\delta_{0}=2 \pi \rho_{s 0} / L_{\|}$, the dynamical plasma beta $\beta_{0}$ defined above, the electron to ion mass and temperature ratios $\mu:=m_{e} / M_{i}$, the normalised parallel resistivity $\eta_{\| 0}:=0.51 \frac{R_{0}}{\tau_{e 0} c_{s 0}} \frac{m_{e}}{M_{i}}$ and normalised parallel electron heat conductivities $\chi_{\| e 0}:=3.15 \frac{\tau_{e 0} c_{s 0}}{R_{0}} \frac{M_{i}}{m_{e}}$, where $\tau_{e 0}$ is the collision time evaluated at reference.

No separation of variables between background and fluctuation has been made and the dependency of the resistivity and heat conductivity on density and temperature has been kept. Moreover, the dependency on the density in the polarization term of the vorticity equation (3) is kept, i.e. the Boussinesq Approximation (BS) has not been applied. In this sense the model is global. The model conserves energy, apart from the fact that we have neglected advection by the polarisation velocity. This has been shown to have only a negligible effect on the conservation of energy [37].

Bohm sheath boundary conditions $[38,39]$ are applied at the ends of the box :

$$
\begin{aligned}
u_{\|} & = \pm \sqrt{T_{e}}, \quad j_{\|}= \pm n \sqrt{T_{e}}\left[1-\exp \left(\Lambda-\frac{\phi}{T_{e}}\right)\right] \\
\partial_{z} T_{e} & =\mp \frac{\gamma_{e}}{\left(\chi_{\| e 0} T_{e}^{5 / 2}\right)} T_{e} n u_{\|},
\end{aligned}
$$

where the lower sign denotes the boundary at $z=0$ and the upper $\operatorname{sign}$ at $z=2 \pi . \Lambda \approx 0.5 \ln \frac{M_{i}}{2 \pi m_{e}}$ is the sheath floating potential and $\gamma_{e}$ an effective sheath transmission factor. In the $x$-direction homogeneous Neumann boundary condition are applied to all fields except for the electrostatic potential, which is set to $\phi=\Lambda T_{e}$, and the electromagnetic potential, which is set to $A_{\|}=0$. 


\subsection{Boussinesq approximation}

The Boussinesq approximation (BS) is widely used in the plasma turbulence community as it simplifies the numerical treatment significantly. It is based on the assumption that the fluctuations of the density are small relative to the background. However, the BS can be problematic for several reasons:

Firstly, its assumption is factually not fulfilled for SOL conditions, where turbulence is typically intermittent with large fluctuation levels. Although, some studies indicate that the BS has finally only a small quantitative effect [40, 29], this cannot be taken for granted under all conditions. Therefore, we address this issue systematically, and investigate the impact of the Boussinesq approximation on blob propagation in the inertial and sheath limited regime over a wide amplitude range in section 3.3.

Secondly, models which make use of the BS are not global in a strict sense, but hybrid. The full density is e.g. kept in the continuity equation (2) but assumptions on it are made in the polarisation term of the vorticity equation (3). Such varying assumptions in a coupled system of equations can lead to inconsistencies, and different forms of the Boussinesq approximation can be found. In a non-conservative form [41, 23, 42] the spatial and temporal dependence of the density is retained:

$$
\nabla \cdot\left(n \mathbf{u}_{p o l}\right) \rightarrow n \nabla \cdot \mathbf{u}_{p o l},
$$

where $\mathbf{u}_{\text {pol }}=-\left(\frac{d}{d t}+u_{\|} \partial_{z}\right) \nabla_{\perp} \phi$ is the polarisation velocity. It is obvious that this form of the BS breaks the total divergence property of the quasi-neutrality condition $(\nabla \cdot \mathbf{J}=0)$, which the vorticity equation (3) actually represents. According to our experience this non-conservative form of the BS lead to highly unstable conditions for closed field line configurations that often crashed our simulations [37]. On the other hand, taking the density out of the divergence with a constant value, as e.g. used in $[26,25,28]$, preserves the total divergence property and worked generally much better in our experience, although it seems less accurate at first sight. Therefore, our studies will consider in the following only this conservative form of the BS.

\section{Blob dynamics}

In this section, the basic blob dynamics, derived from a simple 2D model, are presented. This helps to understand the polarization mechanism and the convection of the blobs in the SOL. The extension to a 3D thermal model is discussed afterwards.

The basic mechanism can be described on the basis of the vorticity equation (3), neglecting parallel advection and assuming unfavourable curvature:

$$
\nabla_{\perp} \cdot\left[n \frac{d}{d t} \nabla_{\perp} \phi\right]=\partial_{z} j_{\|}-2 \partial_{y} p_{e}
$$

Currents are induced in the perpendicular direction by the ion polarization, which describes the collective plasma motion according to the vorticity and the diamagnetic plasma drift, which arises due to the curvature of the magnetic field. The diamagnetic drift acts as a source for the vorticity (last term on right

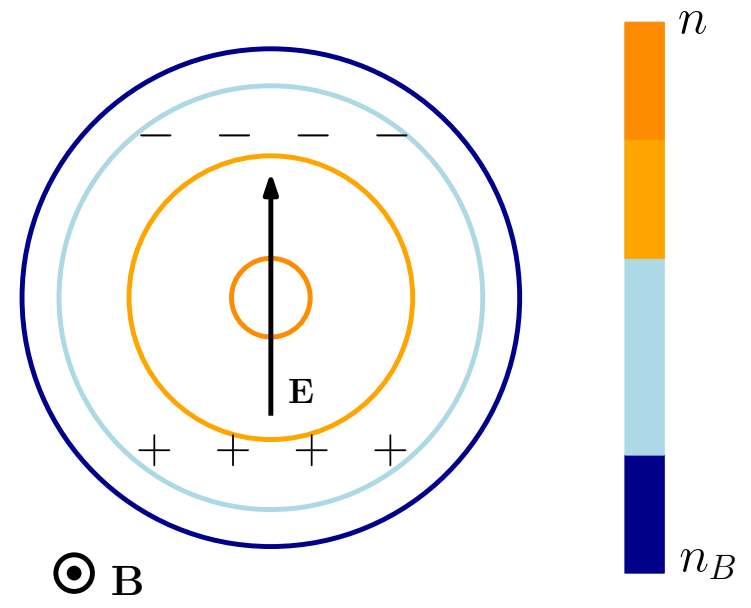

Figure 2: The blob is polarized due to the interchange drive. An electric field arises which is perpendicular to $\mathbf{B}$ and the curvature, causing the $E \times B$ advection of the entire structure. Contours show areas with equal density ranging from the background density $n_{B}$ to the maximum blob density $\mathrm{n}$.

hand side of Eq. (11)), interchanging high density plasma with low density plasma [8]. The diamagnetic drift polarizes the plasma due to the sign dependence for oppositely charged particles. The Gaussian density structure shown in Fig. 2 is polarized due to the interchange drive in Eq. (11), which consequently produces an electric field perpendicular to the magnetic field. This causes an $E \times B$-advection of the entire structure. The blob dynamics depends highly on the balancing of the induced current. The last term on the right hand side of Eq. (11) can be balanced by the inertial term (inertial regime) on the left hand side or by the parallel current, which is limited by the sheath current (sheath limited regime) at the limiter plates. There is extensive theoretical work on scaling laws for the maximum blob velocity as cited in the introduction. We refer here to $[11,43]$, where not only the width but also the amplitude of the blob is taken into account for the scaling law. Given the blob width $\delta_{\perp}$ and its relative amplitude $A_{p}=\Delta p_{e} / p_{e B}$, with $p_{e B}$ the background pressure and $\Delta p_{e}=p_{e}-p_{e B}$ the blob amplitude, the scaling law is:

$$
v_{f}^{I} \propto \sqrt{A_{p} \delta_{\perp}}
$$

in the inertial regime, and:

$$
v_{f}^{S} \propto \frac{T_{e S}^{3 / 2}}{\delta_{\perp}^{2}} \frac{\Delta p_{e}}{p_{e S}},
$$

in the sheath limited regime, where the subscript $S$ denotes that quantity has to be evaluated at the sheath. The sheath limited and inertial regime are separated by a critical scale $[6,44]$ (unnormalised):

$$
\delta_{*}=\rho_{s 0}\left(\frac{L_{\|}^{2}}{\rho_{s 0} R}\right)^{1 / 5}
$$

where the polarisation term and the parallel current term balance both the interchange drive term in Eq. (11) at a comparable order of magnitude. 


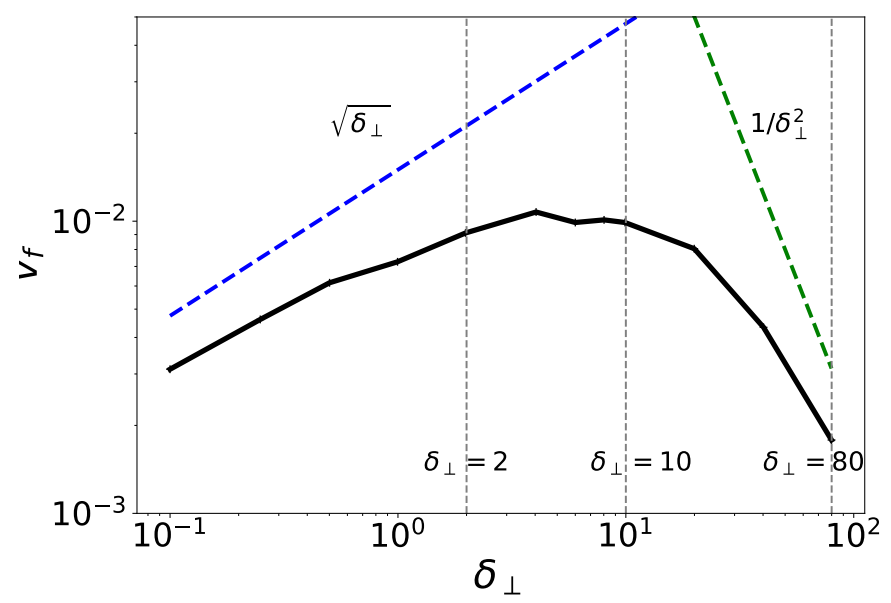

Figure 3: Maximum blob velocity as function of blob width $\delta_{\perp}$. Below the critical scale $\delta_{*} \approx 10$ the blob velocity scales according to the inertial scaling (Eq. (12)) and above according to the sheath limited scaling (Eq. (13)).

The scalings of Eqs. (12) and (13) will be compared against our simulation results in the following sections. Our simulations are based on the following parameters: $R_{0}=1.65 \mathrm{~m}, L_{\|}=$ $8.25 \mathrm{~m}, T_{e 0}=7 \mathrm{eV}, n_{0}=1.0 \cdot 10^{19} \mathrm{~m}^{-3}, B_{0}=1.5 \mathrm{~T}, \Lambda=3, \gamma_{e}=$ 0 and $z_{0}=0$. The simulations are initialised with a 3D Gaussian blob:

$$
n(x, y, z, 0)=n_{B}+\Delta n \exp \left(-\frac{x^{2}+y^{2}}{2 \delta_{\perp}^{2}}\right) \cdot \exp \left(-\frac{(z-\pi)^{2}}{2 L_{\|}^{2}}\right)
$$

and the same for the temperature $T_{e}$. This results in the critical blob width $\delta_{*} \sim 10$ in terms of $\rho_{s}=2.5 \cdot 10^{-4} \mathrm{~m}$, where the inertial and sheath currents are in balance.

\subsection{Scaling with the width}

In this section, the scaling laws for the radial width of the blob in the inertial regime according to Eq. (12) and in the sheath limited regime according to Eq. (13) are verified. For this purpose the radial widths $\delta_{\perp}=$ $[0.1,0.25,0.4,1,2,4,6,8,10,20,40,80]$ are scanned. The perpendicular resolution is set in correlation to the blob width, i.e. $\Delta x=\Delta y=\delta_{\perp} / 10$, and the parallel resolution is $\Delta z=2 \pi / 32$. The logarithm of the maximum values of the velocities $v_{f}$ is plotted against the logarithm of the radial width of the blob in Fig. 3. Below the critical scale $\delta_{*}$ the expected inertial behavior is obtained, where the maximum velocity increases with the radial width $v_{f} \propto \sqrt{\delta_{\perp}}$. The transition to the sheath limited regime appears at the critical blob size $\delta_{\perp} \approx \delta_{*}=10$. In the sheath limited regime the velocity decreases according to the sheath limited scaling $v_{f} \propto \delta_{\perp}^{-2}$. Therefore, the full transition from the inertial regime to the sheath limited regime is successfully recovered within GRILLIX simulations.

\subsection{Scaling with the amplitude}

The scaling laws with respect to the amplitude of the blob are verified. For this purpose the radial widths $\delta_{\perp}=[2,10,80]$ (left, middle and right column in Fig. 4) are chosen, covering inertial, intermediate and sheath limited regime. A constant background density $n_{B}=1$ and electron temperature $T_{e B}=1$ are chosen with varying blob amplitudes in density $\Delta n$ and electron temperature $\Delta T_{e}$.

Firstly (top row of Fig. 4), only the density amplitude is varied with $\Delta T_{e}=1$ fixed. In the inertial regime $\left(\delta_{\perp}=2\right)$ the expected scaling of $v_{f} \propto \sqrt{\Delta n}$ is obtained. A slightly weaker than linear scaling is obtained for $\delta_{\perp}=80$, which is in agreement with the sheath limited scaling (Eq. 13), as the sheath pressure $p_{e S}$ is only weakly dependent on $\Delta n$ (see discussion at end of this section).

Secondly (middle row of Fig. 4), only the electron temperature amplitude is increased with $\Delta n=1$ fixed. In the case $\delta_{\perp}=2$ we observed the blobs to become quickly unstable, which made the determination of the center of mass very difficult and limited the maximum accessible amplitude to $\Delta T_{e}=5$. At $\delta_{\perp}=80$ a slightly higher than linear scaling with $\Delta T_{e}$ is obtained, which is also in agreement with Eq. (13), as the sheath values $T_{e S}$ and $p_{e S}$ are expected to scale with $\Delta T_{e}$ due to parallel heat conductivity.

Finally (bottom row of Fig. 4), $\Delta n$ and $\Delta T_{e}$ are both increased simultaneously. In the inertial regime $\left(\delta_{\perp}=2\right)$ one would expect now a linear scaling at high amplitudes, which is not observed. The reason for this is the shift of the inertial regime towards the sheath limited regime with increasing temperatures. The parallel conductivity is temperature dependent $\sigma_{\|} \propto T_{e}^{3 / 2}$ which makes the sheath closure more favorable with increasing temperature. This effect is captured thanks to the global nature of GRILLIX, and would not be present if the heat conductivity was uniform. For the sheath limited regime $\left(\delta_{\perp}=80\right)$ it is again necessary to know the sheath values of the quantities in order to obtain the scaling. Due to the parallel heat conductivity $T_{e S}$ correlates strongly with $\Delta T_{e}$, whereas this mechanism is absent for the density and $n_{e S}$ is therefore only weakly correlated with $\Delta n$. A scaling that is slightly stronger than linear is eventually observed.

\subsection{Impact of the Boussinesq approximation}

The blob simulations were also carried out in Boussinesq approximation (BS) in order to investigate its impact systematically, and in contrast to [44] the parallel dynamics is taken into account self-consistently. Current balancing suggests that the impact of BS should depend not only on the amplitude $\Delta n$, but also on the blob regime. In the sheath limited regime, where the inertial term is negligible, the impact of the BS is expected to be weak, as it only enters the inertial term. Indeed, we observe in the sheath limited regime (Fig. 4, right column) only very small differences of at most few percent at very high amplitudes.

Towards the inertial regime (Fig. 4, left and center column) the difference between the full system (FS) and BS becomes more pronounced of around $20 \%$ at blob amplitudes of around $\Delta n, \Delta T_{e} \approx 5$, i.e. still moderate given the high amplitude levels. We recognise also differences between BS and FS even if only $\Delta T_{e}$ is varied, which also holds true in the intermediate regime where the blobs remain still coherent and a clear measurement of the center of mass is possible. As discussed before, towards 

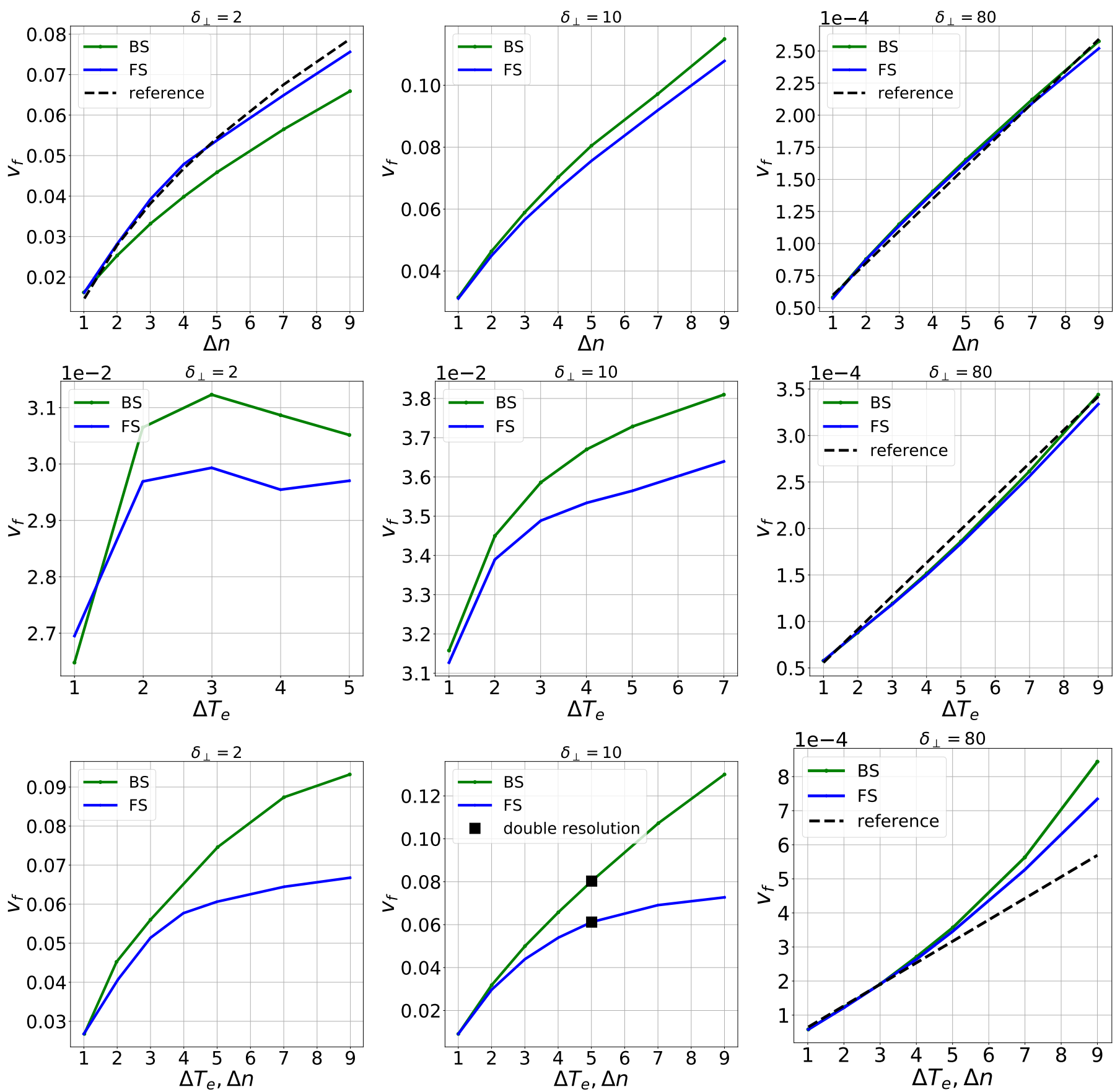

Figure 4: Maximum blob velocity as function of blob amplitude. Left column: for blob of width $\delta_{\perp}=2$ in inertial regime. Middle column: for $\delta_{\perp}=10$ in intermediate regime. Right column: for $\delta_{\perp}=80$ in sheath limited regime. Top row shows results in dependence on density amplitude $\Delta n$, middle row in dependence on temperature amplitude $\Delta T_{e}$ and bottom row in dependence on both varied simultaneously. Results for the full system (FS, blue) and the Boussinesq system (BS, green) are shown. Dashed black lines show some scalings for reference as explained in text. Black points in bottom row and center column show result from simulation with doubled resolution for convergence check. 

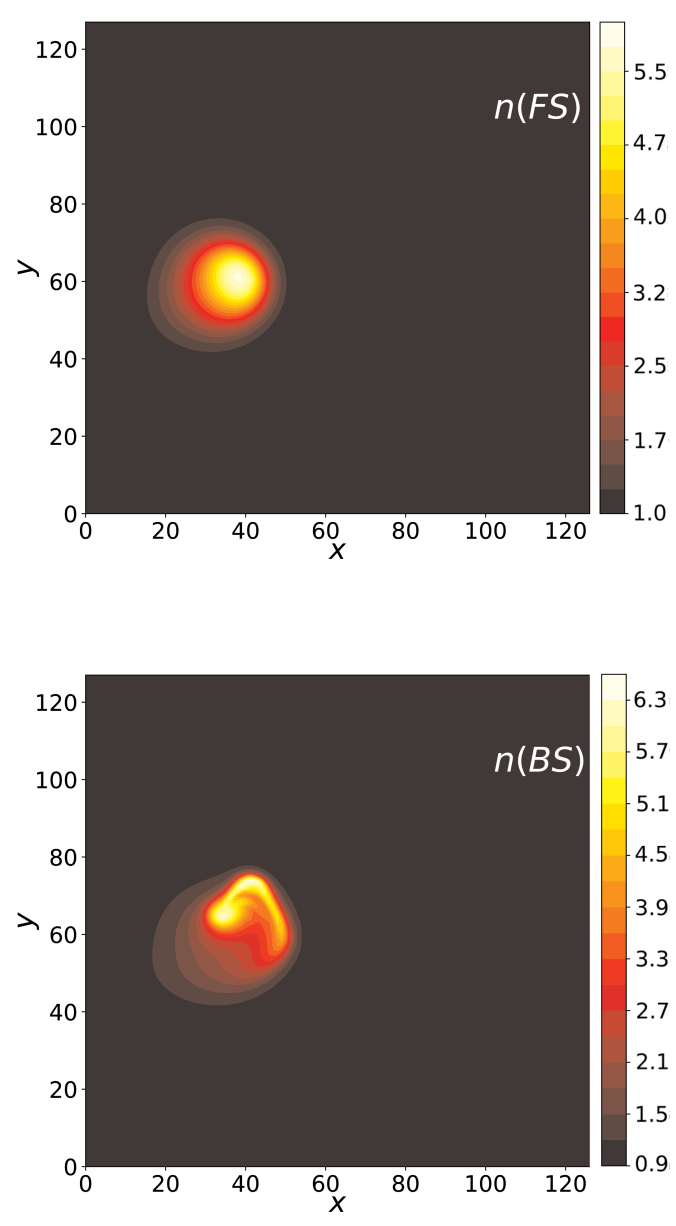

Figure 5: Snapshots of density at $t=0.022$ for blob in intermediate regime $\left(\delta_{\perp}=10\right)$ with amplitude $\Delta n=\Delta T_{e}=5$, simulated with full system (top) and with Boussinesq approximation (bottom).

higher electron temperature the blob transitions from the inertial regime to the sheath limited regime, where the potential starts to follow $\phi \sim \Lambda T_{e}$. Via this effect the temperature enters indirectly also the inertial term and amplifies the difference between full system FS and BS. It is even more pronounced when $\Delta n$ and $\Delta T_{e}$ are increased at the same time. In conclusion, the impact of the BS does not only depend on the density but also the regime is important, which is also subtly influenced by the electron temperature. In previous works (e.g. [44]) this fact was not taken into account self-consistently.

Finally, we highlight in Fig. 5 the qualitative difference between FS and BS. The plot shows a blob with $\delta_{\perp}=10$ and $\Delta n=\Delta T_{e}=5$ at the same time point. In the BS the blob accelerates faster, loses its coherent form and decays, while the blob in FS remains coherent. In order to exclude numerical artefacts this simulation was also performed with the doubled poloidal resolution, yielding the same results.

\section{Large Plasma Device (LAPD)}

\subsection{Setup}

In this section GRILLIX turbulence simulations are compared to the LAPD experiment, a linear machine, which provides a very useful environment for the study of basic plasma turbulence and transport. The axial magnetic field hitting material walls at the end of the device leads to a situation similar to the SOL of a tokamak. Although the densities and temperatures are usually smaller compared to a fusion plasma, the size of the machine allows the growth of many modes in parallel and perpendicular directions, leading to a wide range of turbulent processes relevant for actual fusion devices [34]. The linear plasma column has a length of approximately $L_{\|}=17 \mathrm{~m}$ and a diameter of up to $60 \mathrm{~cm}$. The LAPD plasma parameters are given by the maximum density $n_{0}=2.0 \times 10^{12} \mathrm{~cm}^{-3}$, the electron temperature $T_{e 0}=6 \mathrm{eV}$ and the plasma beta $\beta=1.5 \times 10^{-3}$. The low ion temperature of $T_{i 0}=0.5 \mathrm{eV}$ justifies the cold ion assumption. For the modeling of LAPD plasma the slab geometry is used without curvature terms, a sheath transmission factor of $\gamma_{e}=2.5$ and a floating potential of $\Lambda=3$. The perpendicular domain width spans from $-L_{\perp} / 2$ to $L_{\perp} / 2$ with $L_{\perp}=1.4 \mathrm{~m}$. Density and electron temperature sources are modeled with top-hat shaped profiles $S_{n, T}$ respectively:

$$
S_{n, T}=C_{n, T}\left(1-\tanh \left[\left(r-r_{s}\right) / L_{s}\right]\right) \exp \left(-\lambda_{s} z\right),
$$

with $\lambda_{s}=0.0813$ the decay length of the ionisation front in the parallel direction. The source is radially symmetric $(r=$ $\sqrt{x^{2}+y^{2}}$ ) and has an extent of $r_{s}=20$ decaying sharply with $L_{s}=0.5$. The source rates $C_{n, T}=0.27$ are chosen to match the experiment. A grid with $n_{x}=n_{y}=128$ and $n_{z}=32$ was used for the simulations in this section.

Previous works on LAPD used an electrostatic model with the non-conservative BS [35, 45] or a superficial geometry neglecting the parallel boundaries [46]. Here, simulation results of GRILLIX, based on a truly global and electromagnetic model, are compared to experimental data from LAPD.

\subsection{Blob generation}

There are three relevant plasma instabilities in LAPD that may be present simultaneously [46, 47, 48], the resistive driftwave instability (DW), the rotational interchange instability (RII) and the Kelvin-Helmholtz instability (KHI).

Driven by the density gradient $L_{n} \sim(d(\log n) / d r)^{-1} \mathrm{DW}$ are a common phenomenon in magnetised plasmas [49]. They are characterised by a small phase difference between density and the electrostatic potential $\left(\delta_{n \phi} \lesssim \pi / 4\right)$. The latter two of the mentioned instabilities are caused by the background radial electric field. Due to the sheath boundaries there is a radially varying background electrostatic potential $\phi_{0}(r) \sim \Lambda\left\langle T_{e}\right\rangle$, where $\left\langle T_{e}\right\rangle$ is the average electron temperature. The drive for both instabilities can be understood by considering the $E \times B$ advection term in the vorticity equation (3). Employing a polar coordinate system $r=\sqrt{x^{2}+y^{2}}, \tan \theta=y / x$, applying for clarity the Boussinesq approximation and keeping only terms that 
are linear in the potential fluctuations $\phi=\phi_{0}(r)+\tilde{\phi}$ yields:

$$
\begin{aligned}
\mathbf{v}_{E} \cdot \nabla \nabla_{\perp}^{2} \phi & \approx-\frac{\delta}{r}\left\{\left[\tilde{\phi}, \nabla_{\perp}^{2} \phi_{0}\right]_{r, \theta}+\left[\phi_{0}, \nabla_{\perp}^{2} \tilde{\phi}\right]_{r, \theta}\right\} \\
& =-\frac{\delta}{r}\left\{\left[\tilde{\phi}, \frac{\phi_{0}^{\prime}}{r}\right]_{r, \theta}+\left[\tilde{\phi}, \phi_{0}^{\prime \prime}\right]_{r, \theta}+\left[\phi_{0}, \nabla_{\perp} \tilde{\phi}\right]_{r, \theta}\right\}
\end{aligned}
$$

where we introduced the Jacobi bracket $[f, g]_{r, \theta}=$ $\left(\partial_{r} f \partial_{\theta} g-\partial_{\theta} f \partial_{r} g\right)$. The last two terms in Eq. (17) drive the KHI due to a radially sheared flow. The KHI is discussed for linear machines in detail in $[35,45,50]$ and exhibits a phase difference of $\delta_{n \phi} \approx \pi / 2$ to $\pi[48,51]$. The first term on the right hand side of Eq. (17) is only present due to the rotation of the cylindric plasma column itself. It represents a centrifugal force and is destabilising for a radially decreasing background potential $d\left(\log \phi_{0}\right) / d r<0$. The RII exhibits a phase difference between $\delta_{n \phi} \approx \pi / 4$ to $\pi / 2[48,47]$ and typically mode number of $m=1$ or $m=2$ [51]. Snapshots from a full 3D turbulence simulation are shown in Fig. 6, where the initial plasma column decays into turbulence. We observe the azimuthal rotation $v_{\Theta}$ of the plasma column that is caused by the sheath boundary condition at the plates of the machine $\phi \sim \Lambda\left\langle T_{e}\right\rangle$.

In the following, we will investigate the dominant mechanism for blob generation in LAPD. In linear devices like LAPD, CSDX, and VINETA the blob-ejection process is preceded by an acceleration of an $m=1$ mode $[52,53,54,55]$. Unlike in tokamak plasmas, there is no curvature in linear devices causing a polarisation of the blob. Therefore, another mechanism for blob generation and propagation must exist, namely either of the above mentioned instabilities. We investigate in Fig. 7 two distinctive blobs (dashed boxes) at a particular time point. These blobs are traced back in time until the onset of the blob generation, which is at $t=76.5$ for the blob in box 1 and at $t=77.3$ for the blob in box 3 . At these particular time points, we perform a Morlet wavelet transform [56, 57, 58, 59] in azimuthal direction of the density and potential at the source boundary. This allows a space and wavenumber resolved calculation of the of the local cross-coherence and phase between density and potential. The result of the wavelet analysis is shown in Fig. 8 for $t=76.5$. We observe a strong crosscoherence between $n$ and $\phi$ and a phase shift around $\pm \pi / 2$ at the azimuthal angle $\Theta=\pi / 2$ and $\Theta=3 / 2 \pi$ with $m=1$. The phaseshift around $\pi / 2$ creates a positive perturbation (box 1), which is disconnected from the rest of the plasma, due to the rotation. This results in the generation of a blob. The phase-shift around $-\pi / 2$ creates a negative perturbation (box 2 ), which pushes the plasma inwards. Another similar event with a phase difference close to $\pi / 2$ is seen at $t=77.3$ (box 3 ), which also generates a blob. The appearance of the cross-phase of around $\pi / 2$ between the density and potential at the mode number $m=1$ indicates that the mechanism for blob generation in LAPD is the RII. Eventually, this is a qualitative discussion at a particular time point, but we observed this mechanism also at any further times analysed in our simulation, i.e. each time a blob is created, there was the discussed cross-phase relation between $n$ and $\phi$ around $m=1$.

\subsection{Validation}

The experimental data from $[35,36]$ is compared to the result of the GRILLIX simulation. The considered quantities to be compared are taken at at $z=\pi$ and averaged over time.

The density profile, fluctuation level and skewness are shown in Fig. 9. The density profile is overall in good agreement, with deviations to the experiment becoming pronounced towards the source region $x<0.28 \mathrm{~m}$. The fluctuation amplitude $\delta n=\sqrt{\left\langle n^{2}-\langle n\rangle^{2}\right\rangle}$ is plotted normalized to the maximum value of the density $n_{\max }$ and its course agrees very well with the experiment peaking at the source edge, where the strongest gradients are present. However the absolute fluctuation level obtained from GRILLIX is around a factor of 2 too high. The skewness $\gamma n=\left\langle n^{3}-\langle n\rangle^{3}\right\rangle / \delta n^{3 / 2}$ is a statistical measure for the presence of holes and blobs. A negative skewness is an indicator for the presence of holes, while a positive skewness indicates blobs. The remarkable dip in the experimental data at $x \approx 0.35 \mathrm{~m}$ is -much less pronounced however- only recovered by GRILLIX without the Boussinesq approximation. Otherwise, the overall trend is captured with GRILLIX, i.e. the source region is dominated by holes whereas the source free region is dominated by blobs. Finally, the probability density function (PDF) taken at the source edge and the power spectrum (calculated by squaring the discrete Fourier transform of the density in a volume at the source edge $x=0.25 m-x=0.28 m$, and using a Hanning window) are shown in Fig. 10 and show an excellent agreement between GRILLIX and the experimental data. The PDF has a nearly Gaussian shape indicating a pure uncorrelated behavior of the plasma in the gradient region.

The LAPD simulation run with BS show only very minor quantitative differences to the FS in the statistical averages. At most, the FS matches better the experimental data for the power spectrum at high frequencies and qualitatively recovers the dip in the skewness at $x=0.35$. This indicates that the BS might be misleading under extreme conditions. As an example we show snapshots of the FS and BS simulation at the initial transition to turbulence at $t=4.5$ in Fig. 11, where very steep gradients are present. Under these conditions we indeed observe a qualitative difference between FS and BS.

In conclusion, GRILLIX is able to reproduce the experimental data qualitatively and to a large extent also quantitatively, where deviations to the experiment may have several reasons. Firstly, there are uncertainties from the experimental side, e.g. the profiles of the density differ between [36] and [35] although similar plasma parameters were used. Secondly, the question arises if the experimental data shows general results. As was pointed out in [35], also other values for e.g. the fluctuation level have been measured. Finally, the effect of neutral gas has not yet been taken into account in GRILLIX, which could be potentially important in LAPD $[60,36,61]$.

\section{Conclusions}

The global fluid turbulence code GRILLIX was applied to (quasi)-linear geometry. Although a description of fusion plasmas in linear geometry would be an oversimplification, it is yet 

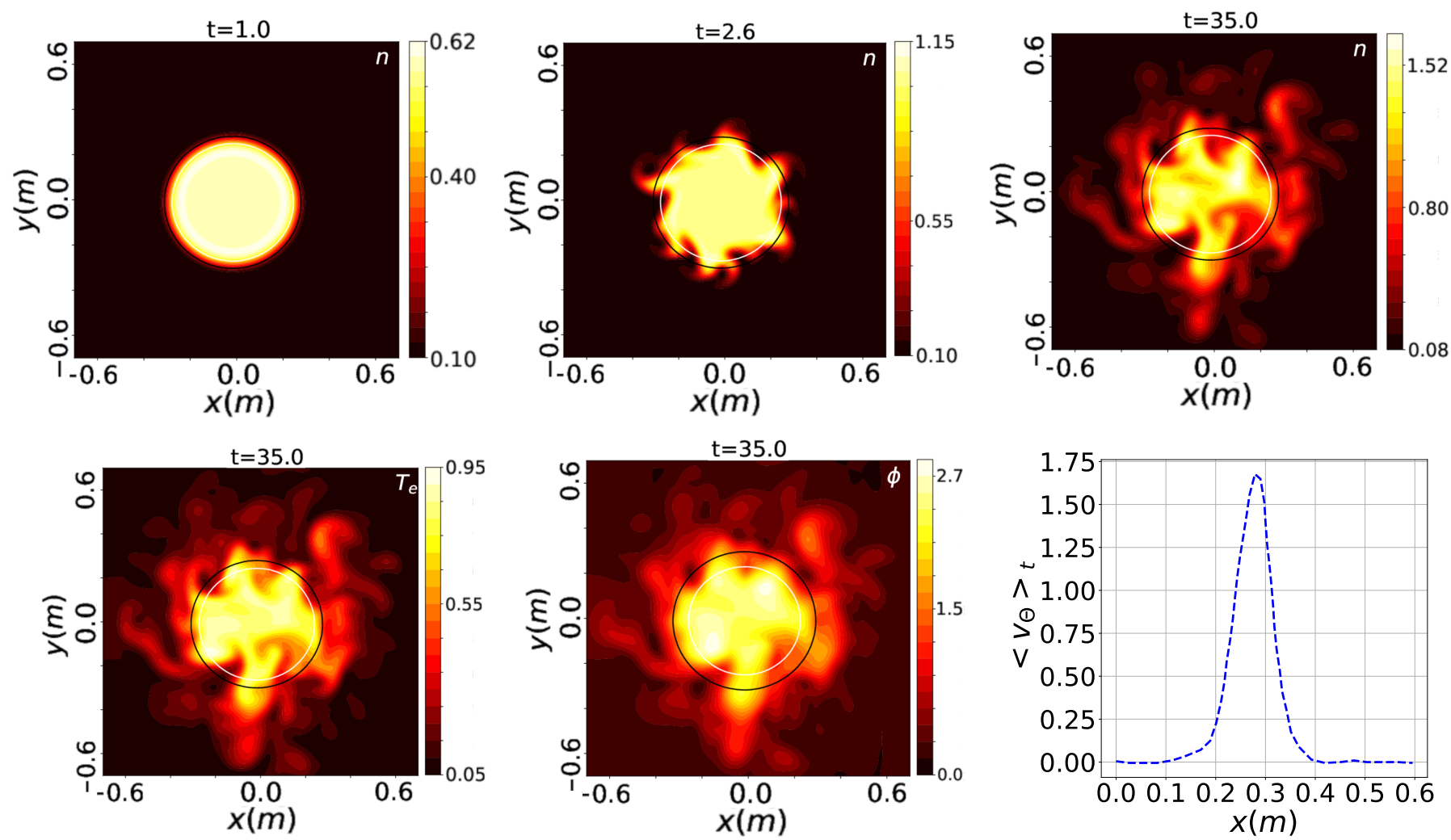

Figure 6: Overview over LAPD simulation: Snapshots of density during initial state (top left), at transition to turbulence (top middle) and in saturated state (top right) and bottom row for $T_{e}$ and $\phi$. White circle indicates top of the source (black circle source boundary). Bottom right: Normalised poloidal flow profile in saturated regime averaged over time.

very useful for the study of basic common phenomena and for thorough code verification and validation, which is often difficult in complex geometries.

The focus of this work was on the study of blob dynamics, and the scaling laws for the inertial regime, Eq. (12), and for the sheath limited regime, Eq. (13), were successfully verified with GRILLIX. We investigated systematically the effect of the Boussinsq approximation and found that not only the fluctuation amplitude in density is important, but also the blob regime affects the validity of the Boussinesq approximation, which is also influenced by temperature. This is important as it points out that the validity of the routinely applied Boussinesq approximation cannot generally be taken for granted. Via the Morlet wavelet analysis we investigated the origin of blobs in the linear device LAPD. During the blob/hole formation we found $m=1$ modes with cross phases of $\delta_{n \phi} \approx \pm \pi / 2$ between density and electrostatic potential. This points on the rotational interchange and Kelvin-Helmoltz instabilities as candidates for the blob drive mechanism.

Finally, we validated our simulation results against the LAPD experiment, where GRILLIX is able to reproduce experimental data of LAPD qualitatively and to a large extent also quantitatively. We found that the Boussinesq approximation has only a very minor impact on statistical averages, but can be significant in extreme conditions, when e.g. steep gradients appear.

\section{Acknowledgements}

This work has been carried out within the framework of the EUROfusion Consortium and has received funding from the Euratom research and training program 2014-2018 and 20192020 under grant agreement No 633053. The views and opinions expressed herein do not necessarily reflect those of the European Commission.

[1] G. Y. Antar, S. I. Krasheninnikov, P. Devynck, R. P. Doerner, E. M. Hollmann, S. C. Boedo, J A Luckhardt, C. R. W, Phys. Rev. Lett. 87 (2001) 065001.

[2] G. Y. Antar, G. Counsell, Y. Yu, B. Lambombard, P. Devynck, Phys. Plasmas 10 (2003) 419.

[3] B. D. Dudson, N. Ben Ayed, A. Kirk, H. R. Wilson, G. Counsell, X. Xu, M. Umansky, P. B. Snyder, Lloyd, Plasma Phys. Control. Fusion 50 (2008) 124012.

[4] A. J. Thornton, G. Fishpool, A. Kirk, Plasma Phys. Control. Fusion 57 (2015) 115010.

[5] D. A. Russell, J. R. Myra, D. A. DIppolito, Phys. Plasmas 16 (2009) 122304.

[6] S. I. Krasheninnikov, D. A. D'Ippolito, J. R. Myra, J. Plasma Physics 74 (2008) 679.

[7] D. A. D'Ippolito, J. R. Myra, S. J. Zweben, Phys. Plasmas 18 (2011) 060501.

[8] O. E. Garcia, Plasma Fusion Res. 4 (2009) 019.

[9] S. I. Krasheninnikov, Phys. Lett. 283 (2001) 368

[10] O. E. Garcia, N. H. Bian, W. Fundamenski, Phys. Plasmas 13 (2006) 082309.

[11] J. Omotani, F. Militello, L. Easy, N. R. Walkden, Plasma Phys. Control. Fusion 58 (2015) 014030

[12] C. Theiler, I. Furno, P. Ricci, A. Fasoli, B. Labit, S. H. Mueller, G. Plyushchev, Phys. Rev. Lett. 103 (2009) 065001. 

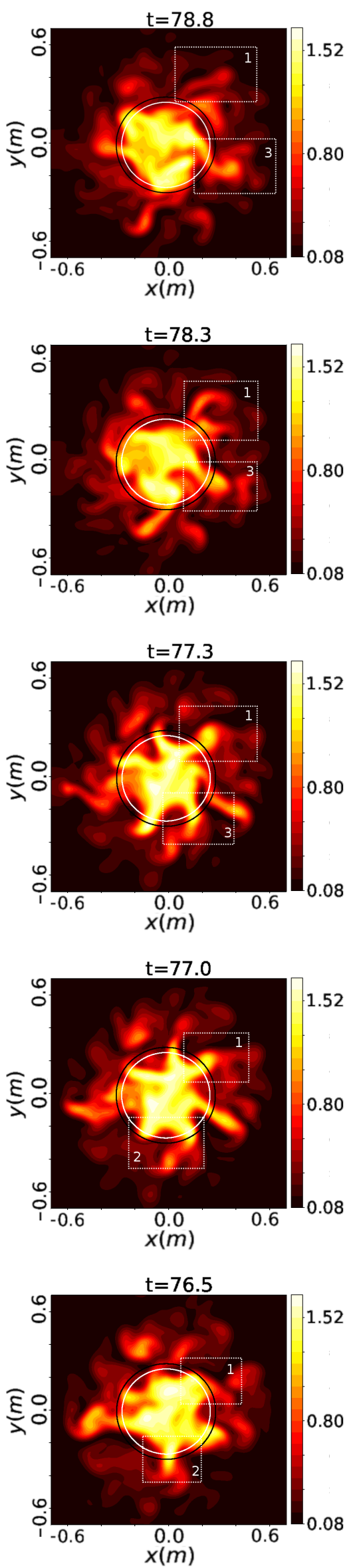

a)

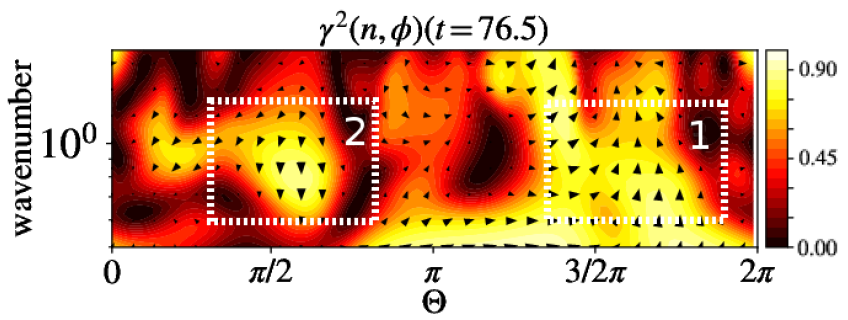

b)

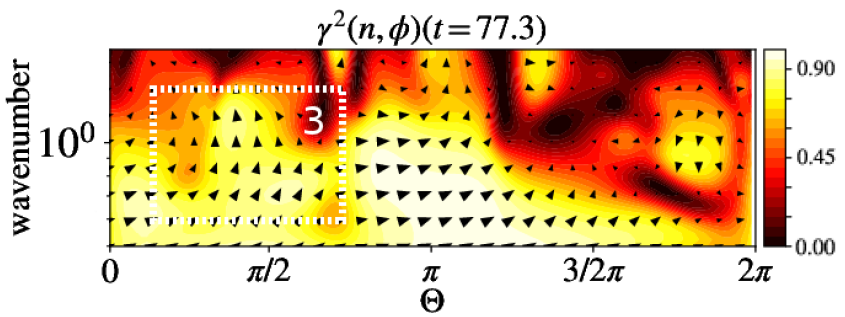

Figure 8: Wavelet squared cross-coherence between $n$ and $\phi$. The orientation of the arrows relative to the positive horizontal axis indicates the phase difference $\delta_{n \phi}$. a) At $t=76.5$ and modenumber $m=1$ a phase difference of $\delta_{n \phi}=\pi / 2$ is seen at the azimuthal position $\Theta=3 / 2 \pi$ (box 1) triggering a blob and a phase difference of $\delta_{n \phi}=-\pi / 2$ at $\Theta=\pi / 2$ (box 2) triggering a hole. b) At $t=77.3$ a phase shift around $\pi / 2$ is observed at $\Theta=3 / 2 \pi$, triggering another blob.

[13] O. E. Garcia, V. Naulin, A. H. Nielsen, J. J. Rasmussen, Phys. Rev. Lett. $92(2004) 165003$

[14] J. R. Myra, D. A. DIppolito, S. I. Krasheninnikov, Phys. Plasmas 11 (2004) 4267.

[15] F. Militello, W. Fundamenski, V. Naulin, A. H. Nielsen, Plasma Phys. Control. Fusion 54 (2012) 095011.

[16] F. Militello, V. Naulin, A. H. Nielsen, Plasma Phys. Control. Fusion 55 (2013) 074010.

[17] J. R. Angus, M. V. Umansky, S. I. Krasheninnikov, Phys. Rev. Lett. 108 (2012) 215002.

[18] F. Easy, L Militello, J. Omotani, N. R. Walkden, B. Dudson, Phys. Plasmas 23 (2016) 012512.

[19] N. R. Walkden, B. D. Dudson, L. Easy, G. Fishpool, J. T. Omotani, Nucl. Fusion 55 (2015) 113022.

[20] P. Ricci, F. Riva, C. Theiler, A. Fasoli, I. Furno, F. D. Halpern, J. Loizu, Phys. Plasmas 22 (2015) 055704.

[21] P. Tamain, H. Bufferand, G. Ciraolo, C. Colin, P. Ghendrih, F. Schwander, E. Serre, Contrib. Plasma. Phys. 54 (2014) 555.

[22] F. Militello, B. Dudson, L. Easy, A. Kirk, P. Naylor, Plasma Phys. Control. Fusion 59 (2017) 125013.

[23] P. Ricci, F. D. Halpern, S. Jolliet, J. Loizu, A. Mosetto, A. Fasoli, I. Furno, C. Theiler, Plasma Phys. Contr. F. 54 (2012) 124047.

[24] F. D. Halpern, P. Ricci, S. Jolliet, J. Loizu, J. Morales, A. Mosetto, F. Musil, F. Riva, T. M. Tran, C. Wersal, J. Comput. Phys. 315 (2016) 388.

[25] B. D. Dudson, J. Leddy, Plasma Phys. Contr. F. 59 (2017) 054010.

[26] P. Tamain, H. Bufferand, G. Ciraolo, C. Colin, D. Galassi, P. Ghendrih, F. Schwander, E. Serre, J. Comput. Phys. 321 (2016) 606

[27] B. Zhu, M. Francisquez, B. N. Rogers, Comput. Phys. Commun. 232 (2018) 46.

[28] A. Stegmeir, D. Coster, A. Ross, O. Maj, K. Lackner, E. Poli, Plasma Phys. Contr. F. 60 (2018) 035005.

[29] A. Stegmeir, A. Ross, T. Body, M. Francisquez, W. Zholobenko, D. Coster, O. Maj, P. Manz, F. Jenko, B. N. Rogers, K. S. Kang, Plasma Phys. Contr. F. 26 (2019) 052517.

[30] A. Zeiler, J. F. Drake, B. Rogers, Phys. Plasmas 4 (1997) 2134.

[31] S. I. Braginskii, Transport processes in a plasma, in: A. M. A. Leontovich (Ed.), Reviews of Plasma Physics, Vol. 1, Consultants Bureau, 1965.

[32] F. Hariri, M. Ottaviani, Comput. Phys. Commun. 184 (2013) 2419. 
a)

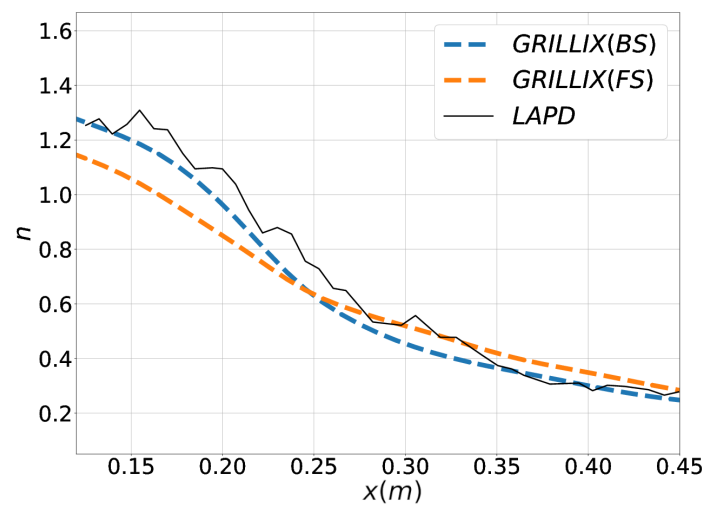

b)

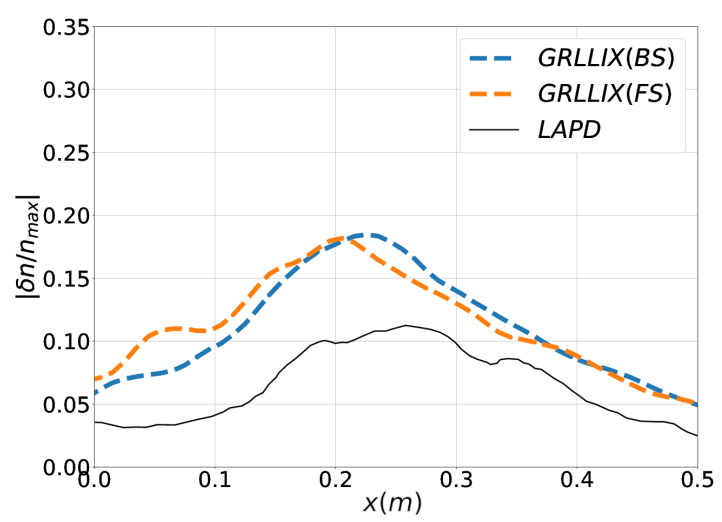

c)

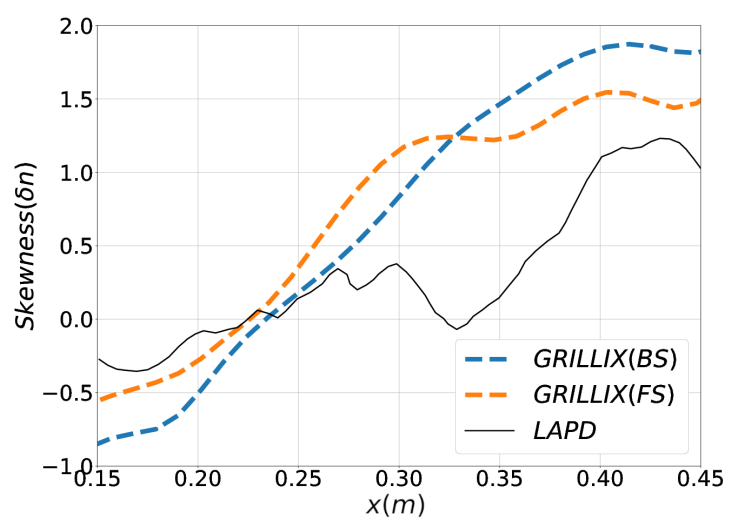

Figure 9: Comparsion of GRILLIX simulation to experimental data (black lines). Density profile (a), fluctuation level (b) and skewness (c) at $z=\pi$ and $y=0$. Simulation results are shown for GRILLIX run with (BS) and without (FS) Boussinesq approximation. a)

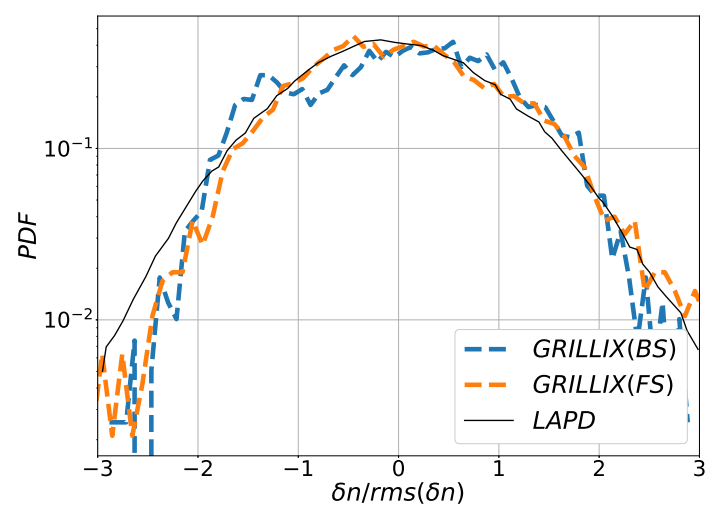

b)

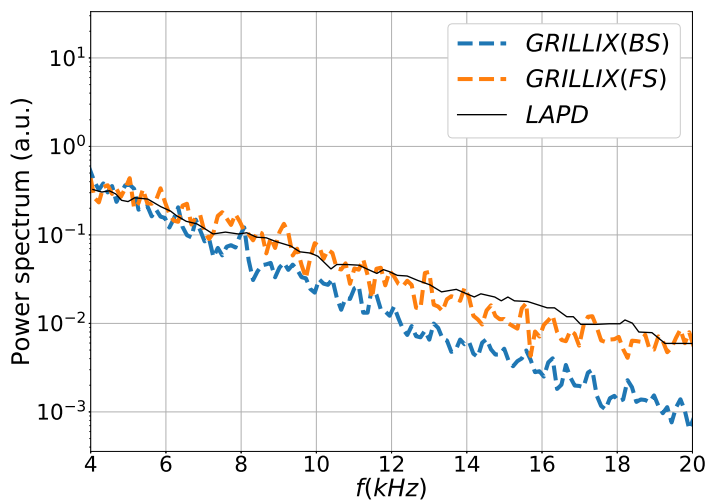

Figure 10: a) Probability density function b) Power distribution Both quantities at $z=\pi$ and source boundary. 

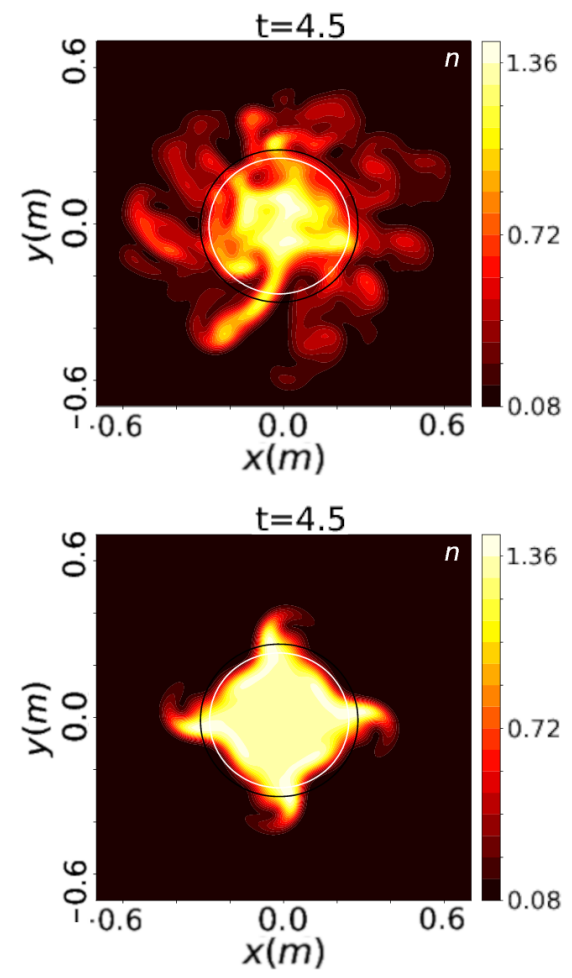

Figure 11: Comparison of density in the FS (top) and the BS (bottom) at the transition to turbulence. The transition to tubulence in FS is shown in Fig. 6 (top middle).

[33] A. Stegmeir, D. Coster, O. Maj, K. Hallatschek, K. Lackner, Comput. Phys. Commun. 198 (2016) 139.

[34] W. Gekelman, P. Pribyl, J. Lucky, M. Drandell, D. Leneman, J. Maggs, S. Vincena, B. Van Compernolle, S. K. P. Tripathi, G. Morales, T. A. Carter, A. Wand, T. DeHass, Rev. Sci. Instrum. 87 (2016) 025105.

[35] D. M. Fisher, B. N. Rogers, G. D. Rossi, D. S. Guice, T. A. Carter, Phys. Plasmas 22 (2015) 092121.

[36] T. A. Carter, J. E. Maggs, Phys. Plasmas 16 (2009) 012304.

[37] A. Ross, A. Stegmeir, D. Coster, Contrib. Plasma. Phys. 58 (2018) 484.

[38] P. C. Stangeby, The Plasma Boundary of Magnetic Fusion Devices, Plasma Physics Series, Institute of Physics Publishing, 2000.

[39] J. Loizu, P. Ricci, F. D. Halpern, S. Jolliet, Phys. Plasmas 19 (2012) 122307.

[40] J. Morales, B. Frei, F. Halpern, F. Musil, P. Paruta, P. Ricci, F. Riva, M. Siffert, C. Wersal, The impact of the Boussinesq approximation on plasma turbulence in the scrape-off layer, 43rd EPS Conference on Plasma Physics, Leuven (2016).

[41] J. R. Angus, S. I. Krasheninnikov, Phys. Plasmas 21 (2014) 112504.

[42] P. Paruta, P. Ricci, , F. Riva, C. Wersal, C. Beadle, B. Frei, Phys. Plasmas 25 (2018) 112301.

[43] A. Ross, Extension of GRILLIX: Towards a global fluid turbulence code for realistic magnetic geometries, Ph.D. thesis, Technical University of Munich (2018).

[44] G. Q. Yu, S. I. Krasheninnikov, P. N. Guzdar, Phys. Plasmas 13 (2006) 042508.

[45] D. M. Fisher, Rogers, Phys. Plasmas 24 (2017) 022303.

[46] P. Popovich, M. V. Umansky, T. A. Carter, B. Friedman, Phys. Plasmas 17 (2010) 102107.

[47] D. A. Schaffner, Study of Flow, Turbulence and Transport on the Large Plasma Device, Ph.D. thesis, University of California (2013).

[48] T. R. Desjardins, M. Gilmore, Phys. Plasmas 13 (2016) 055710.

[49] F. Jeffrey, Plasma Physics and Fusion Energy, Cambridge University Press, 2007.

[50] B. Rogers, P. Ricci, Phys. Rev. Lett. 104 (2010) 225002.

[51] D. L. Jassby, Phys. Fluids 15 (1972) 1590.
[52] T. Windisch, O. Grulke, T. Klinger, Phys. Plasmas 13 (2006) 122303.

[53] G. Y. Antar, J. H. Yu, G. R. Tynan, Phys. Plasmas 14 (2007) 022301.

[54] D. C. Pace, M. Shi, J. E. Maggs, G. J. Morales, T. A. Carter, Phys. Plasmas 15 (2008) 122304.

[55] P. Manz, M. Xu, S. H. Mueller, N. Fedorczak, S. C. Thakur, J. H. Yu, G. R. Tynan, Phys. Rev. Lett. 107 (2011) 195004.

[56] C. H. K. Chen, S. Boldyrev, Astrophys. J 842 (2017) 122.

[57] S. Lion, O. Alexandrova, A. Zaslavsky, Astrophys. J 824 (2016) 47.

[58] A. Grinsted, J. C. Moore, A. Jevrejeva, Nonlin. Proceses Geophys. 11 (2004) 561.

[59] D. Groselj, Fully Kinetic Simulations of Microscale Turbulence in Space and Astrophysical Plasmas, Ph.D. thesis, LMU (2018).

[60] J. Leddy, B. Dudson, H. Willett, Nucl. Mater. Energy 12 (2017) 994.

[61] S. I. Krasheninnikov, A. I. Smolyakov, Phys. Plasmas 10 (2010) 3020 